\title{
Absorption cooling sources atmospheric emissions decrease by implementation of simple algorithm for limiting temperature of cooling water
}

\author{
Krzysztof Wojdyga ${ }^{1, *}$, and Marcin Malicki ${ }^{2}$ \\ ${ }^{1}$ Faculty of Building Services, Hydro and Environmental Engineering of Warsaw University of \\ Technology, ul. Nowowiejska 20, 00-653 Warsaw, Poland \\ ${ }^{2}$ New Energy Transfer, ul. Domowa 6, 02-913 Warsaw, Poland
}

\begin{abstract}
Constant strive to improve the energy efficiency forces carrying out activities aimed at reduction of energy consumption hence decreasing amount of contamination emissions to atmosphere. Cooling demand, both for air-conditioning and process cooling, plays an increasingly important role in the balance of Polish electricity generation and distribution system in summer. During recent years' demand for electricity during summer months has been steadily and significantly increasing leading to deficits of energy availability during particularly hot periods. This causes growing importance and interest in trigeneration power generation sources and heat recovery systems producing chilled water. Key component of such system is thermally driven chiller, mostly absorption, based on lithium-bromide and water mixture. Absorption cooling systems also exist in Poland as stand-alone systems, supplied with heating from various sources, generated solely for them or recovered as waste or useless energy. The publication presents a simple algorithm, designed to reduce the amount of heat for the supply of absorption chillers producing chilled water for the purposes of air conditioning by reducing the temperature of the cooling water, and its impact on decreasing emissions of harmful substances into the atmosphere. Scale of environmental advantages has been rated for specific sources what enabled evaluation and estimation of simple algorithm implementation to sources existing nationally.
\end{abstract}

\section{Introduction}

The strategic objective of Poland's energy and environmental policy is to improve energy efficiency in the area of energy production, supply and use [1]. The energy efficiency target is to reduce energy consumption by $20 \%$ compared to the 2020 projections [2]. Energy

\footnotetext{
* Corresponding author: Krzysztof.Wojdyga@is.pw.edu.pl
} 
efficiency issues are at the heart of the European Union, considered to be the most effective method of reducing primary energy consumption, leading directly to reducing emissions to the atmosphere and, consequently, improving air quality. An essential element of energy efficiency improvement is the maximizing usage of heat that has so far been discharged into the atmosphere and convert it into useful energy. For example waste heat can be used for the production of cold. In the world, cooling systems are responsible for from $16 \%$ to $50 \%$ of electricity consumption [3], with more than $40 \%$ share of energy consumption in the residential and service sector [4]. This leads directly to reaching peak demand for electricity in the summer, when the power of the national electricity system is the smallest. This situation can cause local power deficits, examples of which have appeared more than once in Poland. In spite of its efforts in the area of energy efficiency, Poland still uses 3.4 times more energy to produce goods of the same value than Denmark's most energyefficient economy and 2.2 times more than the EU average [5]. Dominant, in improving energy efficiency, actions focus is on increasing the share of high-efficiency combined heat and power (cogeneration) units that work both for central and local systems. Their part in total energy production should grow in the perspective of a planned $29 \%$ increase in energy consumption by 2030 compared to the 2006 level [6], as a technology capable of achieving efficiency of more than $90 \%$ [7], which is significantly higher than in the case of the split generation [8] and contributing to the reduction of $\mathrm{CO}_{2}$ emissions by supplied with, for example, gas [9] and energy production in the place where it is needed, further reducing transport losses [10]. High-efficiency cogeneration is promoted both in terms of investment and exploitation by means of sectoral, regional and national programs. The technology of combined heat, power and cooling (trigeneration) is particularly important here, enabling the production of all necessary media with the highest efficiency - with the lowest consumption of primary energy.

Over the past decade, due to rising energy carriers prices, environmental awareness, introduced bans on the use of hydrofluorocarbon and chlorofluorocarbon refrigerants combined with increased emphasis on energy efficiency and reduction of emissions, the absorption refrigeration technology returned to the dynamic growth retained in previous years by low prices of electricity. Unlike conventional compressor chillers, absorption chillers can be supplied with low quality energy, which can also be obtained from renewable sources, such as solar radiation, geothermal or industrial waste heat. Additionally, refrigerants used in the absorption chillers were found to be environmentally friendly, as opposed to the refrigerants used in conventional electric compressor chillers [11]. The efficiency of the absorption chillers, understood as the ratio of the useful cooling effect to the energy supplying the unit, is lower than the conventional compressor equipment, but the possibility of supplying them with unused or waste heat results in the primary energy consumption being less than conventional solutions [12]. This has also led to speculation about the design of cooling sources that include not only compressor or absorption aggregates, but also hybrid systems that combine compressor units with absorption units in a single installation [13].

\section{Model}

In absorption chillers, the energy is supplied in the form of heat from the combustion of fuel (e.g. gas, biogas, oil [14]), hot water (e.g. from the district heating network, useless/waste heat from a cogeneration or technological process, solar collectors), steam (e.g. from technological processes, or specially made for powering the appliance) or electrical energy (e.g. by means of a heater). The chilled medium most often is water, its anti-freeze mixture, air or other heat transfer medium. The absorption chiller operates on the principle of using the absorption effect (refrigerant absorption) and the desorption 
(separation of the refrigerant from the solution). Boiling refrigerant absorbs heat to provide a useful cooling effect [15]. The absorber and desorber system in the absorption chiller is called a chemical compressor and corresponds to the functionality of an electrically powered compressor in conventional chillers.

The efficiency of the absorption chiller $(\zeta)$ is defined as the ratio of cooling capacity (Qc) to desorption heat (Qd) at constant temperature of desorption (Td) and evaporation (Tp). The numerical value of $\zeta$ is easy to calculate because it depends on the temperature. Based on steady evaporation and desorption temperatures, the efficiency of the absorption chiller circuit is increased when the condensation temperature is lowered. This temperature is mainly dependent on the cooling water temperature (in most commercially available units cooling water flows through the condenser and the absorber in parallel to provide the same cooling water temperature for both exchangers).

In order to assess the effect of decreasing the cooling water temperature, a model of the influence of the cooling water temperature on the consumption of hot water of the lithium-bromide absorption chiller [16] was developed. Hot water consumption for all working points have been gathered from chiller manufacturer (for constant and decreased water temperature), validated by experiment, and reflected in Microsoft Excel spreadsheet. Afterword's water consumption data has been combined with the data collected, at hourly intervals, from the actual operation of the air-conditioning of the office building. The result of the complete model operation is the hourly hot water requirement for the lithiumbromide absorption chiller working for the office building's air conditioning needs in the annual cycle with standard and decreased water temperature. Under Polish climate conditions, the peak demand for air-conditioning occurs from June to August. This is due to the occurrence of the highest values of annual temperature and the highest values of solar radiation in this period. In addition to monthly changes, the demand for cooling also changes hourly or daily, putting the cooling source in constantly variable operational conditions - most of the time significantly lower than nominal. In practice, the demand for air-conditioning cooling depends directly on the outdoor temperature and solar radiation. This is also a key factor affecting the temperature of the outlet water from the cooling system (e.g. cooling towers or drycoolers). When the cooling demand for air conditioning (i.e. the absorption chiller) is reduced, the cooling systems can provide a lower than the nominal cooling water temperature. This leads to an increase in cooling efficiency at partial loads, and consequently to a reduction in heat demand when the cooling water temperature is reduced. This relation is directly connected with lowering the cooling water temperature [17]. In order to assess the full environmental effect, $\mathrm{CO}_{2}, \mathrm{SO}_{\mathrm{x}} / \mathrm{SO}_{2}, \mathrm{NO}_{\mathrm{x}} / \mathrm{NO}_{2}$ and total suspended particle emissions were calculated for a absorption chiller supplied with heat from a water boiler of less than $5 \mathrm{MW}$ capacity using coal as a fuel. For this, procedure and adequate emission factors for the combustion of fuels made available by the National Center for Balancing and Emissions Management were carried out for each of the compounds. The effect of the model is also the comparison of the heat consumption values for the absorption chiller. Figure 1 presents hot water usage for chiller working with and without algorithm decreasing cooling water temperature throughout model year and Figure 2 during one summer day. 


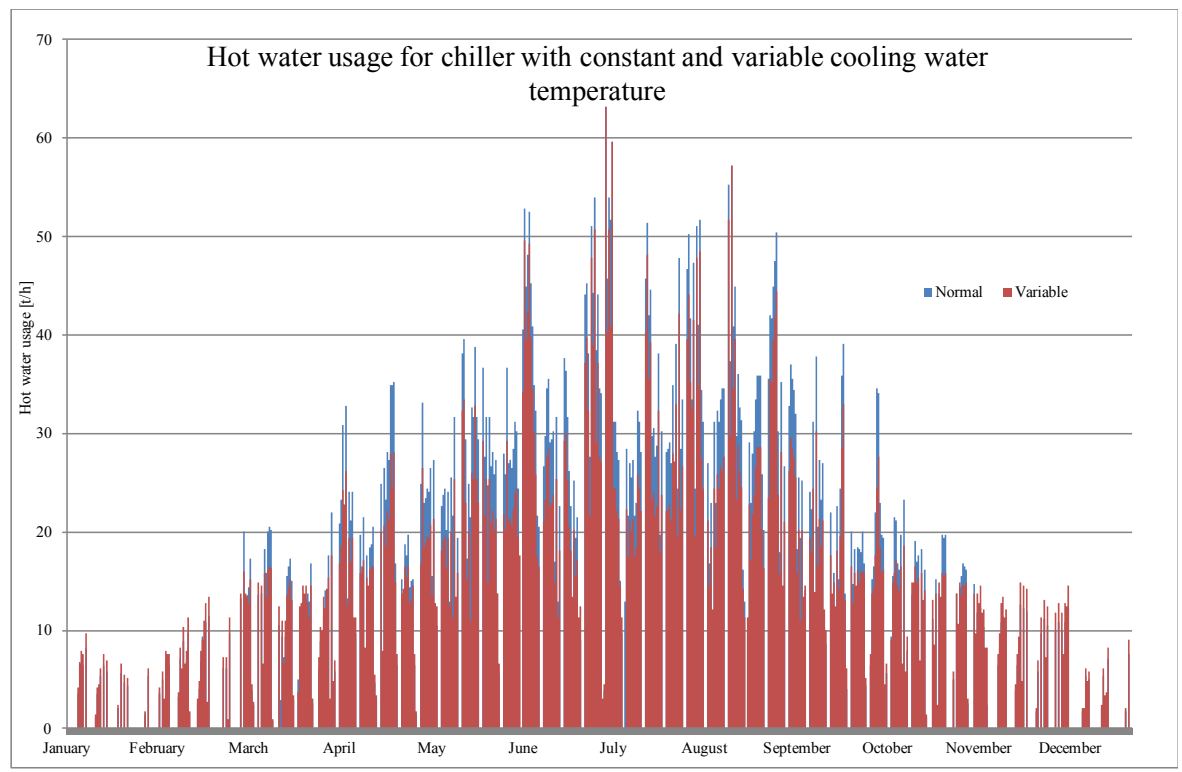

Fig. 1. Absorption chiller hot water usage with normal and variable cooling water temperature during model year.

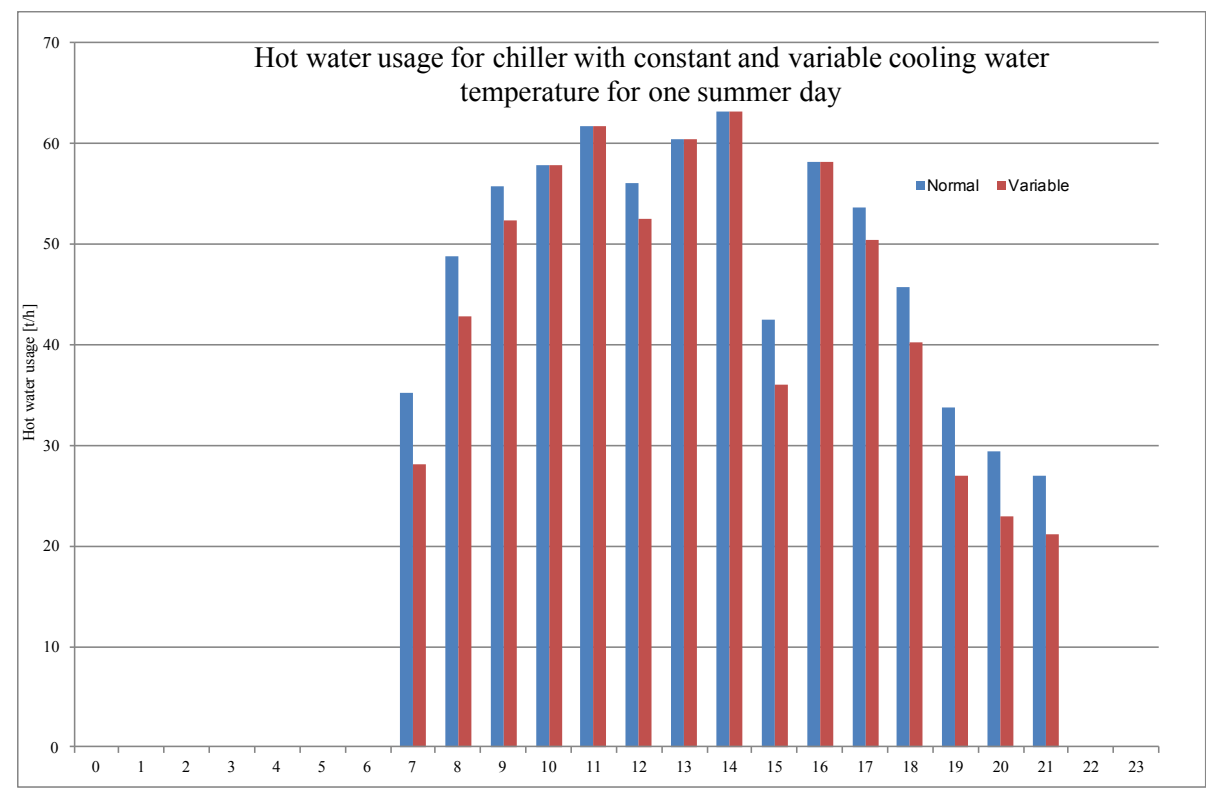

Fig. 2. Absorption chiller hot water usage with normal and variable cooling water temperature during one summer day.

In the analyzed case for $1100 \mathrm{~kW}$ chiller supplied by gas CHP unit, the annual savings are equal to $6800.49 \mathrm{t}$ of hot water at $90 / 70^{\circ} \mathrm{C}(570.56 \mathrm{GJ}$ of heat saved, representing $11.79 \%$ of total heat consumption for cooling purposes), which means $\mathrm{CO}_{2}$ emissions decrease of 35.39 t. For the abovementioned heat source consisting of below $5 \mathrm{MW}$ coal 
boiler, $\mathrm{CO}_{2}$ emissions would be reduced by $57.04 \mathrm{t}, \mathrm{SO}_{\mathrm{x}} / \mathrm{SO}_{2}$ by $465.45 \mathrm{~kg}, \mathrm{NO}_{\mathrm{x}} / \mathrm{NO}_{2}$ by $85.56 \mathrm{~kg}$, and dust by $128.34 \mathrm{~kg}$ per year.

\section{Impact}

To assess scale of environmental advantages of algorithm for cooling water decrease in absorption chillers implementation, cooling sources, including lithium-bromide chillers, have been quantified and separated by medium (eg. hot water, steam) and by primary energy source (gas, coal, biomas). For each energy source primary energy conversion efficiency has been calculated (whenever data was available from the user) or assessed basing on installed equipment. Data about existing installations has been gathered basing on direct contact with exploitation party, publications and commercial materials study. Totally more than 30 installations were analysed from which $23 \%$ were steam chillers and $77 \%$ hot water chillers. More than $44 \%$ of units were supplied with heat from gas fired CHP unit (mainly based on gas engine) and more than $23 \%$ from waste heat or biomass boilers. Rest of the chillers were supplied with heat generated in conventional installations (mainly coal boilers).

\section{Energy source for cooling sources including absorption chillers}

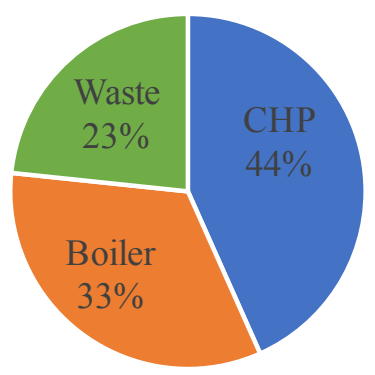

Fig. 3. Energy sources used for energy supply to absorption chillers.

Total heat, for supplying absorption chillers, decrease potential has been calculated for 10200 GJ yearly what corresponds to reduction of $\mathrm{CO}_{2}$ emissions by $603.61 \mathrm{t}, \mathrm{SO}_{\mathrm{x}} / \mathrm{SO}_{2}$ by $3.08 \mathrm{t}, \mathrm{NO}_{\mathrm{x}} / \mathrm{NO}_{2}$ by $566.64 \mathrm{~kg}$ and dust by $849.96 \mathrm{~kg}$ per year. In atmospheric emissions decrease waste heat and biomass installations had been neglected.

Taking into consideration steady absorption chiller market growth and need for energy efficiency improvement yearly new 1 to 3 installations are predicted (market growth by $\sim 5 \%$ is considered) from which one will be supplied with CHP unit, one with waste/biomass and one with heat generated from conventional boiler. This allows to predict that within a decade algorithm implementation could lead to atmospheric emissions decrease by $7016 \mathrm{t}$ of $\mathrm{CO}_{2}, \mathrm{SO}_{\mathrm{x}} / \mathrm{SO}_{2}$ by $35.8 \mathrm{t}, \mathrm{NO}_{\mathrm{x}} / \mathrm{NO}_{2}$ by $6.6 \mathrm{t}$, and dust by $9.9 \mathrm{t}$. 


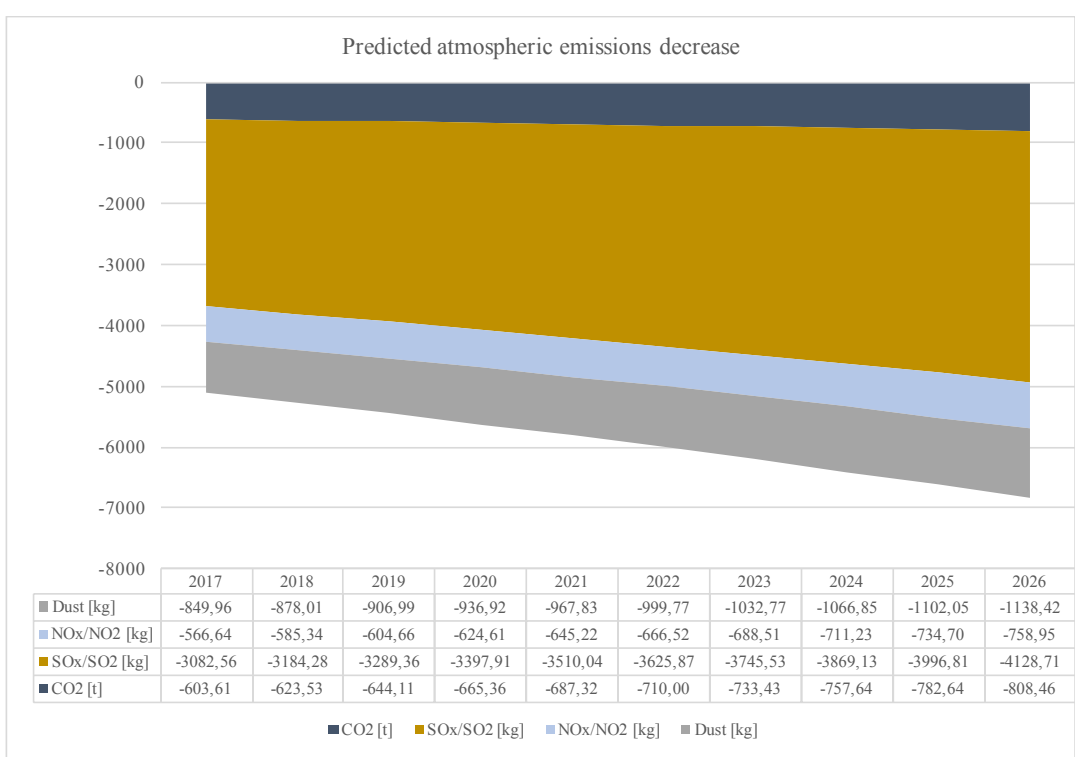

Fig. 4. Predicted atmospheric emissions decrease.

\section{References}

1. National Development Strategy until 2020 as an attachment to Resolution No. 157 of the Council of Ministers of 25 September 2012

2. Announcement of the Minister of Economy of 21 December 2009 on the state energy policy until 2030

3. R. Saidur, H. Masiuki, M. Jamaluddin, Ener. Pol. 35, 1050-1063 (2007)

4. Directive 2002/91/EC "Energy Performance of Buildings", Official Journal of the European Commission, 65-71 (2003)

5. W. Dołęga, Ener. Mark. 2, 24-28 (2014) (in Polish)

6. T. Popławski, Ener. Mark. 1, 13-18 (2014) (in Polish)

7. L. Fu, XL. Zhai, S.G. Shang, Y. Jiang, H. Li, W.W. Yang, Ener. Conv. And Man. 50, 977-82 (2009)

8. A. Marantans, Appl. Therm. Eng. 18, 940-946 (1998)

9. N. Fumo, P. Mago, L. Chamra, Appl. Ener. 86, 2344-50 (2009)

10 J. Ortiga, J.C. Bruno, A. Coronas, Appl. Therm. Eng. 50, 1536-42 (2013)

11. R. Saravanan, M.P. Maiya, Appl. Therm. Eng. 18, 553-68 (1998).

12. F.C. McQuiston, J.D. Parker, Heating, Ventilation and Air conditioning: analysis and design (4th edition, John Wiley and Sons Inc., 1994)

13. M. Meckler, Ener. Eng. 95, 3, 8-13 (1998)

14. W. Kotowski, B. Doliński, Ener. Giga. 6-7 (2013) (in Polish)

15. ASHRE Handbook, Absorption Cooling, Heating, and Refrigeration Equipment (1988)

16. M. Malicki, The influence of chiller parameters change on the trigeneration source efficiency, Ph. D. Thesis (Warsaw University of Technology 2016) (in Polish)

17. H. Yin, An absorption chiller in micro BHCP application: model based design and performance analysis, $\mathrm{Ph}$. D. thesis (Carnegie Mellon University, 2006) 